\title{
Neurovascular Compression Caused by Popliteus Muscle Enlargement Without Discrete Trauma
}

\author{
Kyoung Jin Cho, $\mathrm{MD}^{1}$, Sangkuk Kang, $\mathrm{MD}$, $\mathrm{PhD}^{1}$, Sanghyung Ko, $\mathrm{MD}^{1}$, \\ Junghyun Baek, $\mathrm{MD}^{1}$, Yeongkyun Kim, $\mathrm{MD}^{2}$, Noh Kyoung Park, MD ${ }^{1}$
}

Departments of ${ }^{1}$ Physical Medicine and Rehabilitation, ${ }^{2}$ Vascular Surgery, Sun General Hospital, Daejeon, Korea

Popliteal entrapment syndrome caused by isolated popliteus muscle enlargement is very rare, although its occurrence has been reported after discrete trauma. However, popliteal artery stenosis with combined peroneal and proximal tibial neuropathy caused by popliteus muscle enlargement without preceding trauma has not been reported. A 57-year-old man presented with a tingling sensation and pain in his left calf. He had no previous history of an injury. The symptoms were similar to those of lumbosacral radiculopathy. Calf pain became worse despite treatment, and the inability to flex his toes progressed. Computed tomography angiography and magnetic resonance imaging of the lower extremity showed popliteal artery stenosis caused by popliteus muscle enlargement and surrounding edema. An electrodiagnostic study confirmed combined peroneal and proximal tibial neuropathy at the popliteal fossa. Urgent surgical decompression was performed because of the progressive neurologic deficit and increasing neuropathic pain. The calf pain disappeared immediately after surgery, and he was discharged after the neurologic functions improved.

Keywords Popliteus muscle, Tibial neuropathy, Popliteal fossa

\section{INTRODUCTION}

Popliteal entrapment syndrome presents with vascular or neurogenic symptoms due to the compression of a neurovascular bundle by the surrounding musculoskeletal structure in the popliteal fossa. Entrapment by the tendinous arch of the soleus or gastrocnemius, hematoma after trauma or surgery, and extraneural mass effect have been reported as causes [1]. Popliteal entrapment syndrome caused by isolated popliteus muscle is very rare [2-7]. The condition has been reported after discrete trauma. We report the first case of neurovascular com-

Received July 13, 2015; Accepted September 11, 2015

Corresponding author: Noh Kyoung Park

Department of Physical Medicine and Rehabilitation, Sun General Hospital, 29, Mokjung-ro, Jung-gu, Daejeon 34811, Korea. Tel: +82-42-220-8468, Fax: +82-303-3130-8468, E-mail: nkpark@sunhospital.co.kr

ORCID: Kyoung Jin Cho (http://orcid.org/0000-0001-7690-9067); Sangkuk Kang (http://orcid.org/0000-0003-0563-3280); Sanghyung Ko (http://orcid. org/0000-0001-6651-2533); Junghyun Baek (http://orcid.org/0000-0002-7296-0153); Yeongkyun Kim (http://orcid.org/0000-0001-6333-8568); Noh Kyoung Park (http://orcid.org/0000-0002-6950-5387).

(c) This is an open-access article distributed under the terms of the Creative Commons Attribution Non-Commercial License (http://creativecommons.org/ licenses/by-nc/4.0) which permits unrestricted noncommercial use, distribution, and reproduction in any medium, provided the original work is properly cited. Copyright () 2016 by Korean Academy of Rehabilitation Medicine 
pression caused by popliteus muscle enlargement without preceding trauma.

\section{CASE REPORT}

A 57-year-old male visited our department with a 5-day history of severe left calf pain. He had no significant medication history except for intermittent oral medication for low back pain and right knee pain. He had no history of recent trauma and denied performing any hard work.

At the time of his hospital visit, the left calf was generally soft and not tender to palpation. Left calf pain scored $7 / 10$ in a numeric rating scale (NRS), and there was numbness in the posterolateral aspect of the left calf and sole. Manual muscle testing showed grade-IV strength in the left ankle dorsiflexors, ankle plantar flexors, great toe extensors, and great toe flexors. The result of a straight leg raising test showed $80^{\circ}$ for the right leg and $30^{\circ}$ for the left leg. Tinel's sign was not seen in the popliteal fossa and medial ankle, and tests of knee stability were negative. The ankle-brachial index (ABI) ratio was $0.97 / 0.99$, and pulsation of the lower limb was good, so the probability of vascular origin of the pain was considered to be low. Magnetic resonance imaging (MRI) of the lumbar spine showed herniated intervertebral discs at L4-5 and L3-4, along with central spinal stenosis at L2-3, L3-4, and L4-5. Medication and physical therapy were administered for suspected radiculopathic pain.

On day 5 of hospitalization, He reported increasing left calf pain, with a $9 / 10$ score in NRS. Severe pain in the left foot with weight bearing was noted. The left great toe flexors showed a grade-I strength. Reexamination showed good pulsation of the dorsalis pedis artery and posterior tibial artery with the ankle in neutral position but no pulsation with ankle plantar flexion. The ABI was also reduced markedly at $0.87 / 0.63$ with left ankle plantar flexion. Computed tomography (CT) angiography of the lower extremity showed compression and stenosis of the left popliteal artery due to a mass in the popliteus muscle portion (Fig. 1). MRI of the lower extremity showed edema and enlargement of the left popliteus muscle with increased signal intensity, along with neurovascular compression (Fig. 2). The lateral meniscus and other posterolateral corner structures around the knee were normal. Nerve conduction study (NCS) showed no compound muscle action potential (CMAP) in the left tibial nerve and delayed CMAP latency in the deep peroneal nerve. The amplitude of the sensory nerve action potential was decreased in the left superficial peroneal nerve (Table 1). On the basis of these results, the patient was
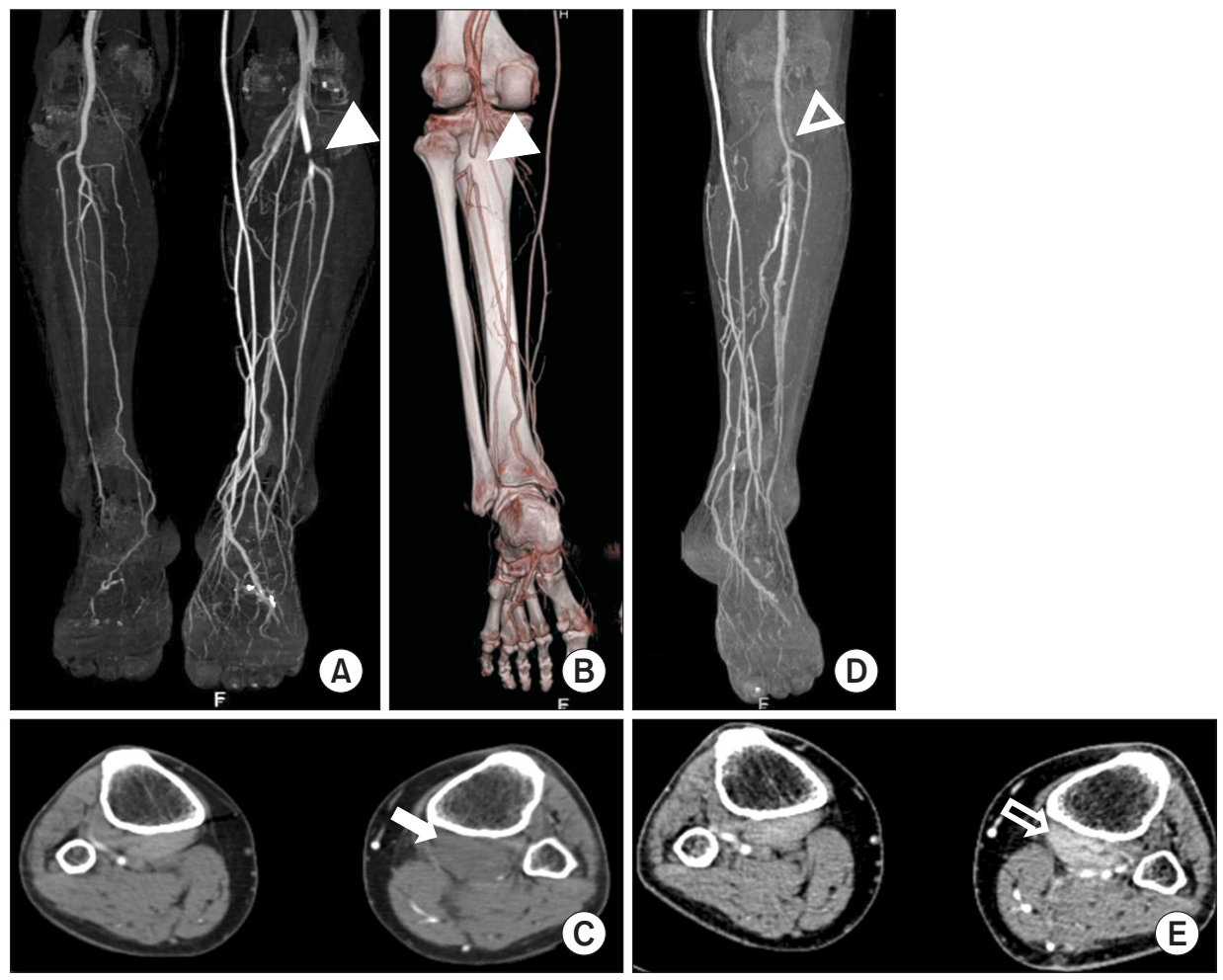

Fig. 1. Computed tomography angiography of lower extremities. (A) Coronal maximal intensity projection and (B) volume rendered image (posterior view) show stenosis and obstruction of the left popliteal artery (arrowheads) and many side branches. (C) Axial image shows a low density mass in the left popliteus muscle portion (arrow). (D, E) At 1 month after operation, obstruction of the popliteal artery was resolved (unfilled arrowhead). The size of the popliteus muscle was decreased (unfilled arrow). 

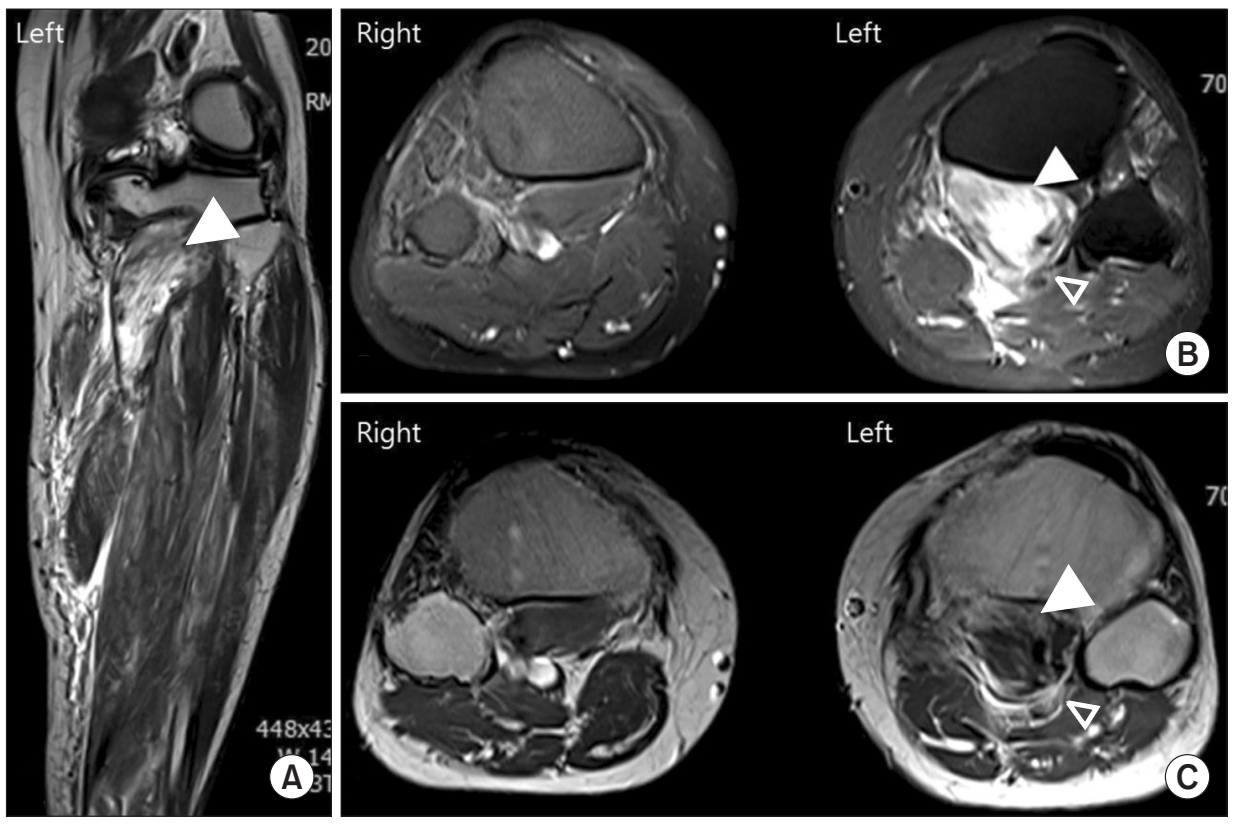

Fig. 2. Magnetic resonance imaging of lower extremities. (A) Coronal T2-weighted image, (B) axial fat-saturated T2-weighted image, and (C) axial T1-weighted image show swelling and enlargement of the left popliteus muscle (arrowheads), along with neurovascular compression (unfilled arrowhead).

Table 1. Results of the nerve conduction study

\begin{tabular}{|c|c|c|c|c|c|c|c|}
\hline \multirow{2}{*}{ Nerve } & \multirow{2}{*}{ Stimulation site } & \multicolumn{3}{|c|}{ Right } & \multicolumn{3}{|c|}{ Left } \\
\hline & & LAT (ms) & AMP & $\mathrm{CV}(\mathrm{m} / \mathrm{s})$ & LAT (ms) & AMP & $\mathrm{CV}(\mathrm{m} / \mathrm{s})$ \\
\hline \multicolumn{8}{|l|}{ Motor } \\
\hline \multirow[t]{3}{*}{ Peroneal (recoding at EDB) } & Ankle & 4.7 & 2.90 & & $5.7^{\mathrm{a})}$ & 2.12 & \\
\hline & Below fibular head & 11.7 & 2.78 & 42 & $12.8^{\mathrm{a})}$ & 1.34 & 42 \\
\hline & Popliteal fossa & 13.9 & 2.72 & 45 & $15.8^{\mathrm{a})}$ & 1.29 & $33^{\text {a) }}$ \\
\hline \multirow[t]{2}{*}{ Peroneal (recoding at TA) } & Below fibular head & 3.5 & 6.10 & & $4.6^{\mathrm{a})}$ & 5.06 & \\
\hline & Popliteal fossa & 5.4 & 5.96 & 53 & $6.5^{\mathrm{a})}$ & 4.90 & 53 \\
\hline \multirow[t]{2}{*}{ Tibial } & Ankle & 4.2 & 15.87 & & $\mathrm{NE}$ & NE & \\
\hline & Popliteal fossa & 14.1 & 10.82 & 41 & $\mathrm{NE}$ & $\mathrm{NE}$ & \\
\hline \multicolumn{8}{|l|}{ Sensory } \\
\hline Superficial peroneal & Lowe leg & 3.3 & 14.28 & & 3.5 & $7.53^{\mathrm{a})}$ & \\
\hline Sural & Lowe leg & 3.3 & 16.11 & & 3.3 & 14.47 & \\
\hline
\end{tabular}

LAT, latency (motor, onset latency; sensory, peak latency); AMP, amplitude (motor, $\mathrm{mV}$; sensory, $\mu \mathrm{V})$; $\mathrm{CV}$, conduction velocity; EDB, extensor digitorum brevis; TA, tibialis anterior; NE, not evoked.

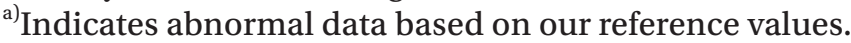

diagnosed with popliteal artery stenosis and combined peroneal and proximal tibial neuropathy due to neurovascular compression by popliteus muscle enlargement.

The patient was transferred to the Department of Vascular Surgery and emergency surgery was performed. The tibial nerve, popliteal artery, and popliteal vein were identified as directly superficial to the popliteus muscle running distally under the firm fibrous arch of the soleus. The neurovascular structures were found to be flattened at the soleus arch. To reduce the mass effect of popliteus muscle, the soleus arch was dissected (Fig. 3). Left calf pain disappeared immediately after surgery. However, muscle weakness and pain on sole persisted in the left lower limb.

Approximately 4 weeks after symptom onset, an electrodiagnostic study was repeated. Findings of the NCS were not different from those of the initial study. The needle electromyography study showed denervation po- 

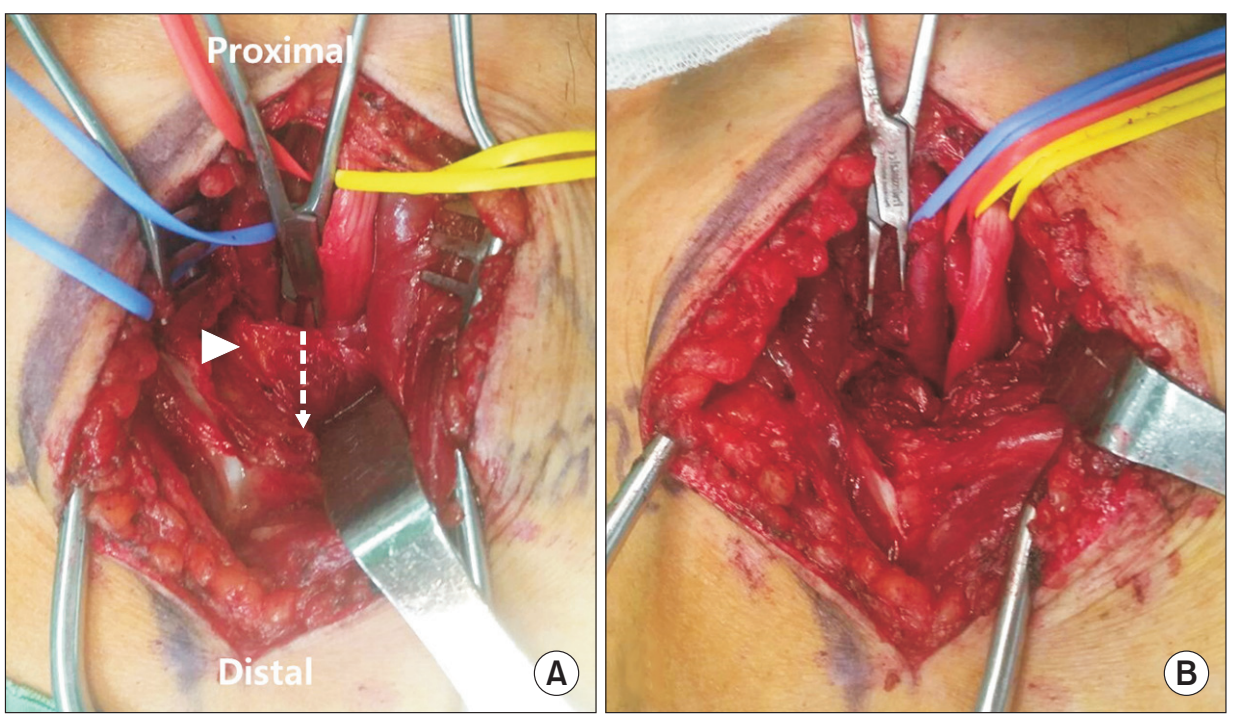

Fig. 3. Left popliteal fossa (yellow, tibial nerve; red, popliteal artery; blue, popliteal vein). (A) Pre-dissected state of the soleus arch (arrowhead) shows compressed tibial nerve and blood vessels. (B) Postdissected state of the soleus arch.

Table 2. Results of needle electromyography

\begin{tabular}{|c|c|c|c|c|}
\hline \multirow{2}{*}{ Muscle } & \multicolumn{2}{|c|}{ ASA } & \multirow{2}{*}{ MUAP } & \multirow{2}{*}{ Interference pattern } \\
\hline & PSW & Fib & & \\
\hline \multicolumn{5}{|l|}{ Bilateral } \\
\hline Paraspinalis, L2-S1 & None & None & Normal & \\
\hline \multicolumn{5}{|l|}{ Left side } \\
\hline Vastus lateralis & None & None & Normal & Full \\
\hline Gluteus medius & None & None & Normal & Full \\
\hline Gluteus maximus & None & None & Normal & Full \\
\hline Tibialis anterior & None & None & Normal & Full \\
\hline Extensor digitorum brevis & None & None & Normal & Full \\
\hline Peroneus longus & None & None & Normal & Full \\
\hline Peroneus brevis & None & None & Normal & Full \\
\hline Gastrocnemius & None & None & Normal & Full \\
\hline Tibialis posterior & + & None & Normal & Reduced \\
\hline Abductor digiti quinti & + & None & Normal & Reduced \\
\hline Abductor hallucis & + & None & Normal & Reduced \\
\hline
\end{tabular}

ASA, abnormal spontaneous activity; PSW, positive sharp wave; Fib, fibrillation potential; MUAP, motor unit action potential.

tentials in the left tibialis posterior, abductor digiti quinti, and abductor hallucis with discrete recruitment patterns (Table 2), confirming combined peroneal and proximal tibial neuropathy around the popliteal space. At 1 month after surgery, CT angiography of the lower extremity was conducted again. The images showed decompression of the popliteal artery, and decreased size of the popliteus muscle (Fig. 1D, 1E).

After 2 months of medical and physical therapy, muscle strength in the left lower extremity improved to almost normal, except for the left great toe flexors. Left sole pain decreased to a $2 / 10$ score in NRS. The patient was left with a partial neurologic deficit with numbness on the left sole and grade-I strength in the left great toe flexors.

\section{DISCUSSION}

The popliteus muscle is a thin, flat, triangular muscle 
that forms the fundus of the popliteal fossa. The muscle runs obliquely across the posterior tibia with a tendinous insertion on the lateral femoral condyle. The tibial nerve, popliteal artery, and popliteal vein course posteriorly to the popliteus muscle, leaving the popliteal fossa distally under the soleus arch $[2-5,8]$. The peroneal nerve descends obliquely along the lateral side of the popliteal fossa to the head of the fibula. The functions of the popliteus muscle are internal rotation of the tibia, unlocking of the knee during early flexion, and static and dynamic stabilization of the lateral side of the knee [4,8]. Popliteus muscle injury can occur in case of a blow to the anteromedial aspect of the knee or sudden external rotation of a hyperextended knee [8]. Most popliteus muscle injuries are associated with other posterolateral corner injuries such as the meniscus and posterior cruciate ligament [2-7].

The patient in this case had not sustained direct trauma but participated in mountain climbing once a week for more than 5 hours a day, and often walked with strain on his left leg due to persistent low back pain and right leg pain. In downhill running or walking, a vector acts to displace the weight-bearing femur forward onto the relatively fixed tibia. The popliteus muscle acts to hold back the femur from forward displacement on the tibia. Therefore, any downhill running or walking may cause increased stress on the popliteus muscle and tendon [8]. High intensity exercise may induce a proteolytic pathway related to muscle damage and trigger inflammatory processes in the muscle [9]. The cause of the patient's condition was difficult to ascertain, but his hobby and walking habits could have resulted in enlargement and edema of the popliteus muscle.

Compared to distal tibial neuropathy in the tarsal tunnel, compression or entrapment of the proximal tibial nerve in the popliteal fossa is less frequently encountered in clinical practice [1]. In particular, among cases of popliteal entrapment syndrome, proximal tibial neuropathy caused by isolated popliteus muscle injury is very rare, with only 6 cases reported so far. In all these cases, proximal tibial neuropathy was preceded by a traumatic event such as injury sustained by jumping, tackling, and falling from a horse, or an event that could have caused knee injury [2-6].

In our case, proximal peroneal neuropathy was concomitant with proximal tibial neuropathy. It is very rare in cases with no direct injury. In 2009, Williams et al. [10] reported simultaneous entrapment of the peroneal and proximal tibial nerves in the popliteal fossa. However, unlike in our case, it was caused not by popliteus muscle.

In 5 of the 6 cases of proximal tibial nerve palsy caused by popliteus muscle injury, there was a normal neurologic recovery after nonsurgical treatment. Progression of lower limb weakness was seen only in one case reported by Bollier et al. [5], for which surgical decompression was performed. In our case, because of obstruction of the popliteal artery and the progression of neurologic deficit, surgical intervention was performed.

Diagnosis of popliteal entrapment syndrome is not easy because the symptoms are similar to those of chronic exertional compartment syndrome, muscle strain, lumbosacral radiculopathy, and spinal stenosis, so careful differentiation is needed. To avoid misdiagnosis, a thorough assessment of the patient's history and physical examination are required. In particular, the physical examination should be performed in a posture that can trigger symptoms such as ankle dorsiflexion/plantar flexion. To avoid unnecessary treatment and the associated disastrous consequences, timely and accurate diagnosis and treatment are needed.

\section{CONFLICT OF INTEREST}

No potential conflict of interest relevant to this article was reported.

\section{REFERENCES}

1. Kim S, Choi JY, Huh YM, Song HT, Lee SA, Kim SM, et al. Role of magnetic resonance imaging in entrapment and compressive neuropathy - what, where, and how to see the peripheral nerves on the musculoskeletal magnetic resonance image: part 1. Overview and lower extremity. Eur Radiol 2007;17:139-49.

2. Geissler WB, Corso SR, Caspari RB. Isolated rupture of the popliteus with posterior tibial nerve palsy. J Bone Joint Surg Br 1992;74:811-3.

3. Bowditch MG, Kay NR. Painful swollen calf due to isolated rupture of popliteus. Injury 1994;25:200-1.

4. de Ruiter GC, Torchia ME, Amrami KK, Spinner RJ. Neurovascular compression following isolated popliteus muscle rupture: a case report. J Surg Orthop Adv 2005;14:129-32. 
5. Bollier M, Ream T, Hodgman G. Isolated popliteus muscle rupture with neurovascular compression requiring surgical decompression. Am J Orthop (Belle Mead NJ) 2010;39:588-91.

6. Dimberg EL, Rubin DI, Ortiguera CJ, Kennelly KD. Popliteus muscle hemorrhage as a rare cause of a proximal tibial neuropathy. J Clin Neurosci 2014;21:520-1.

7. Ortiguera CJ, Bremner BR, Peterson JJ. Popliteus strain causing tibial nerve palsy with a permanent partial deficit: a case report. Am J Sports Med 2006;34:117680.
8. Jadhav SP, More SR, Riascos RF, Lemos DF, Swischuk LE. Comprehensive review of the anatomy, function, and imaging of the popliteus and associated pathologic conditions. Radiographics 2014;34:496-513.

9. Park KS, Lee MG. Effects of unaccustomed downhill running on muscle damage, oxidative stress, and leukocyte apoptosis. J Exerc Nutrition Biochem 2015;19: 55-63.

10. Williams EH, Williams CG, Rosson GD, Dellon AL. Combined peroneal and proximal tibial nerve palsies. Microsurgery 2009;29:259-64. 